PROCEEDINGS OF THE

AMERICAN MATHEMATICAL SOCIETY

Volume 133, Number 1, Pages 91-96

S 0002-9939(04)07626-9

Article electronically published on August 10, 2004

\title{
GROUP AUTOMORPHISMS WITH FEW AND WITH MANY PERIODIC POINTS
}

\author{
THOMAS WARD
}

(Communicated by Michael Handel)

\begin{abstract}
For each $C \in[0, \infty]$ a compact group automorphism $T: X \rightarrow X$ is constructed with the property that

$$
\frac{1}{n} \log \left|\left\{x \in X \mid T^{n}(x)=x\right\}\right| \longrightarrow C
$$

This may be interpreted as a combinatorial analogue of the (still open) problem of whether compact group automorphisms with any given topological entropy exist.
\end{abstract}

\section{INTRODUCTION}

One of the outstanding open problems in the dynamics of compact group automorphisms concerns the existence of group automorphisms with small entropy. Taking the infimum over all compact group automorphisms $T$, and writing $h_{\text {top }}(T)$ for the topological entropy of $T$, is

$$
\inf \left\{h_{\text {top }}(T) \mid h_{\text {top }}(T)>0\right\}>0 ?
$$

As pointed out in [9], the infimum being zero is equivalent to the statement that for any $H \in(0, \infty]$ there is an (ergodic) compact group automorphism with topological entropy $H$. The infimum being positive implies that the set of possible values of topological entropies of compact group automorphisms is countable.

The problem in (11) turns out to be exactly equivalent to Lehmer's Problem (see [9]). In [8], Lehmer associated to any monic polynomial

$$
f(x)=\prod_{i=1}^{d}\left(x-\alpha_{i}\right) \in \mathbb{Z}[x]
$$

the integer sequence defined by $\Delta_{n}(f)=\prod_{i=1}^{d}\left|\alpha_{i}^{n}-1\right|$, and asked how fast this sequence grows. To avoid degeneracies, we assume that $\Delta_{n}(f) \neq 0$ for all $n \geq 1$ (equivalently, $f$ does not vanish on any root of unity). It turns out that the measure of growth $\lim _{n \rightarrow \infty} \frac{\Delta_{n+1}(f)}{\Delta_{n}(f)}$ he used does not always exist, but a consequence of

Received by the editors August 16, 2003.

2000 Mathematics Subject Classification. Primary 37C35, 22D40, 11N13.

Key words and phrases. Group automorphism, periodic points, topological entropy, Lehmer problem.

(C)2004 American Mathematical Society 
Baker's Theorem is that the logarithmic growth rate

$$
m(f)=\lim _{n \rightarrow \infty} \frac{1}{n} \log \Delta_{n}(f)=\sum_{i=1}^{d} \max \left\{\log \left|\alpha_{i}\right|, 0\right\}
$$

exists (see [6, Chap. 1] for the details). Lehmer's problem is the following question: is

$$
\inf \{m(f) \mid f \in \mathbb{Z}[x], m(f)>0\}>0 ?
$$

The equivalence of (11) and (3) is proved in [9]; to see why this equivalence is plausible notice that if $T_{f}$ is the endomorphism of the $d$-torus $\mathbb{T}^{d}$ associated to the companion matrix of the polynomial $f$, then

$$
h_{\mathrm{top}}\left(T_{f}\right)=m(f) .
$$

More is true. The sequence $\left(\Delta_{n}(f)\right)$ counts the periodic points under the automorphism $T_{f}$,

$$
\mathrm{F}_{n}\left(T_{f}\right)=\left|\left\{x \in \mathbb{T}^{d} \mid T_{f}^{n}(x)=x\right\}\right|=\Delta_{n}(f)
$$

whenever either side is finite (ergodicity of $T_{f}$ is equivalent to $\mathrm{F}_{n}\left(T_{f}\right)$ being finite for all $n \geq 1$ ). Thus (2) means that the logarithmic growth rate of the number of periodic points for an ergodic toral automorphism coincides with the topological entropy. Indeed, any continuous map on a compact metric space with sufficiently strong specification properties will have this property. For compact group automorphisms, expansiveness guarantees that the growth rate of periodic points is the topological entropy (this is shown in [10] in greater generality).

Without the assumption of expansiveness or ergodicity, we show that in contrast to the conjectured answer 'yes' to the questions (11) and (3) above, all possible logarithmic growth rates of periodic points arise for compact group automorphisms.

\section{PERIODIC ORBITS}

The examples will be constructed by controlling the growth in the number of orbits of length $n$, so the first step is to relate this to the number of points of period $n$. Given any bijection $T: X \rightarrow X$, define

$$
\mathrm{F}_{n}(T)=\left|\left\{x \in X \mid T^{n}(x)=x\right\}\right|
$$

to be the number of points of period $n$ and

$$
\mathrm{L}_{n}(T)=\left|\left\{x \in X||\left\{T^{j}(x)\right\}_{j \in \mathbb{N}} \mid=n\right\}\right|
$$

to be the number of points with least period $n$. The sequences $\left(\mathrm{F}_{n}(T)\right)$ and $\left(\mathrm{L}_{n}(T)\right)$ determine each other via the relation (17) and its Möbius inversion (see [5, Sect. 11.2] or [15]).

Lemma 1. For $C \in(0, \infty)$,

$$
\lim _{n \rightarrow \infty} \frac{1}{n} \log \mathrm{F}_{n}(T)=C
$$

if and only if

$$
\lim _{n \rightarrow \infty} \frac{1}{n} \log \mathrm{L}_{n}(T)=C
$$


Proof. This is shown in [15. Sect. 4]; a short proof is included here. First notice that

$$
\mathrm{F}_{n}(T)=\sum_{d \mid n} \mathrm{~L}_{d}(T)
$$

If $\frac{1}{n} \log \mathrm{L}_{n}(T) \rightarrow C>0$ then, for $n$ large enough to have $\mathrm{F}_{n}(T)>0$,

$$
\begin{aligned}
\frac{1}{n} \log \mathrm{L}_{n}(T) \leq \frac{1}{n} \log \mathrm{F}_{n}(T) & =\frac{1}{n} \log \left(\sum_{d \mid n} \mathrm{~L}_{d}(T)\right) \\
& \leq \frac{1}{n} \log n+\frac{1}{n} \log \max _{d \mid n}\left\{\mathrm{~L}_{d}(T)\right\} .
\end{aligned}
$$

For each such $n$, choose $\tilde{n}$ so that $\mathrm{L}_{\tilde{n}}(T)=\max _{d \mid n}\left\{\mathrm{~L}_{d}(T)\right\}$ and $\frac{\tilde{n}}{n} \leq 1$. Now $\tilde{n} \rightarrow \infty$ as $n \rightarrow \infty$ since $C>0$. It follows that

$$
\begin{aligned}
\frac{1}{n} \log \mathrm{L}_{n}(T) \leq \frac{1}{n} \log \mathrm{F}_{n}(T) & \leq \frac{1}{n} \log n+\frac{\tilde{n}}{n} \cdot \frac{1}{\tilde{n}} \log \mathrm{L}_{\tilde{n}}(T) \\
& \leq \frac{1}{n} \log n+\frac{1}{\tilde{n}} \log \mathrm{L}_{\tilde{n}}(T) \rightarrow C,
\end{aligned}
$$

so $\frac{1}{n} \log \mathrm{F}_{n}(T) \rightarrow C$.

Now assume that $\frac{1}{n} \log \mathrm{F}_{n}(T) \rightarrow C$. For $r \geq 1$,

$$
\mathrm{F}_{r}(T) \geq \mathrm{L}_{r}(T)=-\sum_{d \mid r, d \neq r} \mathrm{~L}_{d}(T)+\mathrm{F}_{r}(T) \geq \mathrm{F}_{r}(T)-\sum_{d \mid r, d \neq r} \mathrm{~F}_{d}(T) .
$$

Let $R$ be an upper bound for $\left\{\frac{1}{n} \log \mathrm{F}_{n}(T) \mid \mathrm{F}_{n}(T) \neq 0\right\}$; pick $\epsilon \in(0,3 C)$. Choose $N$ so that

$$
r>N \Longrightarrow e^{r(C-\epsilon)} \leq \mathrm{F}_{r}(T) \leq e^{r(C+\epsilon)} .
$$

Then for $r>2 N$,

$$
\begin{aligned}
\mathrm{F}_{r}(T) \geq \mathrm{L}_{r}(T) & \geq \mathrm{F}_{r}(T)-\sum_{n=1}^{N} \mathrm{~F}_{n}(T)-\sum_{n=N+1}^{\lfloor r / 2\rfloor} \mathrm{F}_{n}(T) \\
& \geq \mathrm{F}_{r}(T)-\left(N e^{N R}+(r / 2-N) e^{r(C+\epsilon) / 2}\right) \\
& \geq \mathrm{F}_{r}(T)\left(1-N e^{N R-r(C-\epsilon)}-(r / 2-N) e^{-r(C-3 \epsilon) / 2}\right),
\end{aligned}
$$

and the bracketed expression converges to 1 as $r \rightarrow \infty$. Taking logs and dividing by $r$ gives the result.

Write $\mathbb{F}_{p}=\mathbb{Z} / p \mathbb{Z}$ for the finite field with $p$ elements. For any prime $p$, the field $\mathbb{F}_{p}$ has a primitive root $g$ that generates the cyclic multiplicative group $\mathbb{F}_{p}^{*}$.

Theorem 2. For any $C \in[0, \infty]$, there is a compact group automorphism $T$ with $\mathrm{F}_{n}(T)<\infty$ for all $n$ and with

$$
\frac{1}{n} \log \mathrm{F}_{n}(T) \longrightarrow C
$$

Proof. If $C=0$, then we may take $X$ to be any finite group (more interesting ergodic examples with $X=\widehat{\mathbb{Q}}$ or $\widehat{\mathbb{F}_{p}(t)}$ may be found in [2] and [11]).

Assume now that $C=\infty$. Choose primes $p_{1}, p_{2}, \ldots$ with the following properties:

(1) $\frac{1}{n} \log p_{n} \longrightarrow \infty$, and

(2) $n \mid p_{n}-1$ for all $n \geq 1$. 
This can be done since there are infinitely many primes in the arithmetic progression $(1+n k)_{k \in \mathbb{N}}$ by Dirichlet's theorem. For each $i$, let $g_{i}$ be a primitive root modulo $p_{i}$ and define a map $T_{i}: \mathbb{F}_{p_{i}} \rightarrow \mathbb{F}_{p_{i}}$ by $T_{i}(x)=g_{i}^{\left(p_{i}-1\right) / i} x$ modulo $p_{i}$. Then $\mathrm{F}_{n}\left(T_{i}\right)=p_{i}$ if and only if $i \mid n$; in all other cases, $\mathrm{F}_{n}\left(T_{i}\right)=1$. Define $X=\prod_{i=1}^{\infty} \mathbb{F}_{p_{i}}$ and the automorphism $T=\prod_{i=1}^{\infty} T_{i}$. Then $\mathrm{F}_{n}(T) \geq p_{n}$, so $\frac{1}{n} \log \mathrm{F}_{n}(T) \rightarrow \infty$. Notice that $\mathrm{F}_{n}(T)$ is finite for all $n$.

The argument above uses the fact that arithmetic progressions of the form $(1+n k)_{k \in \mathbb{N}}$ contain infinitely many primes. For $C<\infty$, a related but deeper fact is used. Linnik 12 has shown that the least prime congruent to 1 modulo $n$ is no larger than $n^{A}$ for some absolute constant $A$. Later developments have culminated in Heath-Brown's proof 7 that the bound may be replaced by $B n^{5.5}$ for an explicitly computable constant $B$.

The case $C \in(0, \infty)$ remains. By Lemma 1 it is enough to construct a group automorphism $T$ with $\mathrm{L}_{n}(T)$ close to $e^{n C}$. By Linnik's Theorem, choose primes $p_{1}, p_{2}, \ldots$ with the following properties:

(1) $n \mid p_{n}-1$ for all $n \geq 1$, and

(2) $p_{n} \leq n^{A}$.

Notice that the first property ensures that $p_{n} \rightarrow \infty$ as $n \rightarrow \infty$, and the second property ensures that $\frac{n}{\log p_{n}} \rightarrow \infty$ as $n \rightarrow \infty$. Let $g_{n}$ be a primitive root modulo $p_{n}$, and define

$$
K_{n}=\left\lfloor\frac{n C}{\log p_{n}}\right\rfloor \text { for } n \geq 1,
$$

where $\lfloor x\rfloor$ is the largest integer less than or equal to $x$. For each $n$, let

$$
T_{n}:\left(\mathbb{F}_{p_{n}}\right)^{K_{n}} \rightarrow\left(\mathbb{F}_{p_{n}}\right)^{K_{n}}
$$

be defined by

$$
T_{n}\left(x_{1}, \ldots, x_{K_{n}}\right)=\left(g_{n}^{\left(p_{n}-1\right) / n} x_{1}, \ldots, g_{n}^{\left(p_{n}-1\right) / n} x_{K_{n}}\right) .
$$

Define the compact group by

$$
X=\prod_{i=1}^{\infty}\left(\mathbb{F}_{p_{i}}\right)^{K_{i}},
$$

and the automorphism by $T=\prod_{i=1}^{\infty} T_{i}$.

The map $T_{n}^{j}$ is the identity if and only if $n \mid j$. So $T_{n}^{j}\left(x_{1}, \ldots, x_{K_{n}}\right)=\left(x_{1}, \ldots, x_{K_{n}}\right)$ if and only if $x_{1}=\cdots=x_{K_{n}}=0$ or $n \mid j$. It follows that the number of points of least period $n$ under $T$ is given by

$$
\mathrm{L}_{n}(T)=p_{n}^{K_{n}}-1 .
$$

Thus

$$
\begin{aligned}
\frac{1}{n} \log \mathrm{L}_{n}(T) & =\frac{1}{n} \log \left(p_{n}^{K_{n}}-1\right) \\
& =\frac{K_{n}}{n} \log \left(p_{n}^{K_{n}}-1\right)^{1 / K_{n}} \\
& =\frac{1}{n}\left\lfloor\frac{n C}{\log p_{n}}\right\rfloor \log \left(p_{n}^{K_{n}}-1\right)^{1 / K_{n}} \longrightarrow C
\end{aligned}
$$


as $n \rightarrow \infty$ since $\frac{n}{\log p_{n}} \rightarrow \infty$. It follows by Lemma 1 that

$$
\frac{1}{n} \log \mathrm{F}_{n}(T) \longrightarrow C \text { as } n \rightarrow \infty \text {. }
$$

\section{REMARKS}

(1) The only requirement in the proof is that $K_{n} \rightarrow \infty$, so the same argument allows the construction of group automorphisms with any super-polynomial growth rate, $\frac{\log \mathrm{F}_{n}(T)}{\log n} \longrightarrow \infty$.

(2) A similar infinite product construction is used in [4 to exhibit a group automorphism whose periodic points count the Bernoulli denominators. Moss 13 has obtained results showing when a given realizable divisibility sequence can arise from a group automorphism.

(3) The proof of Theorem 2 constructs a non-ergodic automorphism of a totally disconnected group. Does the same result hold for ergodic automorphisms of connected groups? On connected groups, the type of behaviour seen in automorphisms with few periodic points seems to be $\limsup _{n \rightarrow \infty} \frac{1}{n} \log \mathrm{F}_{n}(T)=h_{\text {top }}(T)$ and $\liminf \operatorname{in}_{n \rightarrow \infty} \frac{1}{n} \log \mathrm{F}_{n}(T)=0$ (see [16], [17]).

(4) The dynamical zeta function associated to a map $T$ with $\mathrm{F}_{n}(T)<\infty$ for all $n \geq 1$ is the formal power series $\exp \sum_{n=1}^{\infty} \frac{z^{n}}{n} \mathrm{~F}_{n}(T)$. Explicit examples of group automorphisms with irrational zeta functions are easy to find (see [2]; it should be noted that the examples of this kind in 3 do not seem to be correct). For values of $C$ that are not reciprocals of algebraic integers, the examples constructed here cannot have rational zeta functions for a purely arithmetic reason (the poles and zeros of a rational zeta function can only occur at reciprocals of algebraic integers by [1, Appendix] or [14, Part VIII, Chap. 4, No. 230]).

\section{REFERENCES}

[1] R. Bowen and O. E. Lanford, III, Zeta functions of restrictions of the shift transformation, Global Analysis (Proc. Sympos. Pure Math., Vol. XIV, Berkeley, Calif., 1968), Amer. Math. Soc., Providence, R.I., 1970, pp. 43-49. MR0271401 (42:6284)

[2] V. Chothi, G. R. Everest, and T. Ward, S-integer dynamical systems: periodic points, J. Reine Angew. Math. 489 (1997), 99-132. MR1461206 (99b:11089)

[3] J. W. England and R. L. Smith, The zeta function of automorphisms of solenoid groups, J. Math. Anal. Appl. 39 (1972), 112-121. MF0307280 (46:6400)

[4] G. Everest, A. J. van der Poorten, Y. Puri, and T. Ward, Integer sequences and periodic points, J. Integer Seq. 5 (2002), no. 2, Article 02.2.3, 10 pp. MR.1938222 (2003j:11014)

[5] G. Everest, A. J. van der Poorten, I. Shparlinksi, and T. Ward, Recurrence Sequences, Mathematical Surveys and Monographs, vol. 104, American Mathematical Society, Providence, RI, 2003. MR 1990179 (2004c:11015)

[6] G. R. Everest and T. Ward, Heights of polynomials and entropy in algebraic dynamics, Springer-Verlag London Ltd., London, 1999. MR1700272 (2000e:11087)

[7] D. R. Heath-Brown, Zero-free regions for Dirichlet L-functions, and the least prime in an arithmetic progression, Proc. London Math. Soc. (3) 64 (1992), no. 2, 265-338. MR1143227 (93a:11075)

[8] D. H. Lehmer, Factorization of certain cyclotomic functions, Ann. of Math. 34 (1933), 461479. MR 1503118

[9] D. Lind, The structure of skew products with ergodic group automorphisms, Israel J. Math. 28 (1977), no. 3, 205-248. MR0460593 (57:586)

[10] D. Lind, K. Schmidt, and T. Ward, Mahler measure and entropy for commuting automorphisms of compact groups, Invent. Math. 101 (1990), no. 3, 593-629. MF 1062797|(92j:22013) 
[11] D. Lind and T. Ward, Automorphisms of solenoids and p-adic entropy, Ergodic Theory Dynam. Systems 8 (1988), no. 3, 411-419. MR0961739 (90a:28031)

[12] U. V. Linnik, On the least prime in an arithmetic progression. I. The basic theorem, Rec. Math. [Mat. Sbornik] N.S. 15(57) (1944), 139-178. MR.0012111 (6:260b)

[13] P. Moss, Algebraic realizability problems, Ph.D. thesis, The University of East Anglia, 2003.

[14] G. Pólya and G. Szegö, Problems and theorems in analysis. Vol. II, Springer-Verlag, New York, 1976, Theory of functions, zeros, polynomials, determinants, number theory, geometry. MR 0465631 (57:5529)

[15] Y. Puri and T. Ward, Arithmetic and growth of periodic orbits, J. Integer Seq. 4 (2001), no. 2, Article 01.2.1, 18 pp. MR.1873399 (2002i:11026)

[16] T. Ward, An uncountable family of group automorphisms, and a typical member, Bull. London Math. Soc. 29 (1997), no. 5, 577-584. MF 1458718 (98k:22028)

[17] _ Almost all S-integer dynamical systems have many periodic points, Ergodic Theory Dynam. Systems 18 (1998), no. 2, 471-486. MR1619569 (99k:58152)

School of Mathematics, University of East Anglia, Norwich NR4 7TJ, United KingDOM

E-mail address: t.ward@uea.ac.uk 